\title{
QoS-aware User Load Management with Beamforming for Energy Efficiency in Wireless Networks
}

\author{
Naveed Ali Kaim Khani, Zhe Chen, and Fuliang Yin
}

\begin{abstract}
To overcome the congestion by load management of cell network, beam breathing based load management scheme is proposed to meet the issue. The proposed energy efficient load management scheme is based on beamforming layer breathing and multiple users handling of single beamforming transmission. The beam breathing is first handled by downlink beamforming transmission power control (breathe in or out) to manage the congestion. Subsequently, a single beam multi-users method for load balancing is discussed. Lastly, the linear equalizers Zero Forcing (ZF) and Minimum Mean Square Error (MMSE) with successive interference cancellation are exploited to recover information from multiplexed transmissions. The proposed scheme is effective to decrease the power consumption, congestion control, load balancing and is also constructive to increase the data rate in wireless communication. Simulation results reveal that the proposed work is quite better than the existing solutions in terms of throughput and energy consumption.
\end{abstract}

Index Terms-Beamforming breathing, load balancing, congestion control, inter-cell interference, energy-efficient network.

\section{INTRODUCTION}

Emerging technologies in cellular networks are growing rapidly to offer higher data rate, lower power consumption and an increasing number of users within the coverage area. Most traditional wireless networks and cellular architectures are interconnected by cell based transmissions, in which base transceiver stations (BTS's) of different cells correspond with their relevant mobile users (MU's), separately [1]. In general, the conventional cell based network has performance issues due to inter-cell interference, spectrum efficiency, limitation of MU's hand-off and congestion control. The beamforming transmission technique can break the limitation of cell-based wireless networks with the ability of user steering based transmission and single frequency reuse. Where, single frequency reuse pattern has boosted the spectrum efficiency due to the frequency band availability for other cells [2]. Therefore, many emerging technologies (e.g. WiMAX, UMTS Long Term Evolution (LTE), LTE-Advance and 5G) have fully adopted beamforming technique for better transmission.

Manuscript received August 3, 2016; revised December 5, 2016. This work was supported by National Natural Science Foundation of China (Nos. 61172107, 61172110), National High Technology Research and Development Program (863 Program) of China (No. 2015AA016306), Major Projects in Liaoning Province Science and Technology Innovation (No. 201302001), and Fundamental Research Funds for the Central Universities of China (No. DUT13LAB06).

All authors are with the School of Information and Communication Engineering, Dalian University of Technology, Dalian, 116023, China (e-mail: Naveedali64@yahoo.fr, zhechen@dlut.edu.cn, flyin@dlut.edu.cn).
Extensive research works are also undergoing to improve the overall performance of beamforming transmission in emerging wireless technologies. There have been plenty of schemes conceiving load balancing issue in wireless and cellular networks with the help of beamforming transmission. The transport layer based congestion control is tackled by transferring users from loaded cells to less congested cells to avoid the link bottleneck for LTE systems in [3]. Additionally, in [4], [5] a joint optimization of beamforming to maximum and association control was investigated to balance the network load across multiple cells by updating beamforming weight vectors to optimize the selection criteria of serving BTs and beamforming policy. In general, channel variation status can be upgraded into beamforming weight vectors within the cell, but the benefit of the proposed work is to exchange the user association between the cells. Furthermore, a load management by internode collaboration and offloading algorithm for interference avoidance scheme is discussed for advanced radio interface technologies such as long-term evolution (LTE)/ Fourth Generation (4G) in [6]. Yan Li. et al. [7] proposed a dynamic massive MIMO beamforming algorithm to reduce inter-cell interference with load management over vertical and horizontal domains of an antenna according to user directions. A load balanced cell sectorization scheme was discussed in [8] through efficiently exploiting the spatial degrees of freedom (DOF) with the help of vertical beamforming techniques and user location determination. In [9], a downlink and uplink dichotomy for the multi-cell case was projected, in which the signal to interference and noise ratio (SINR) restraints for neighbor users are avoided by reducing the overall transmitted power around all base stations. Furthermore, the congestion and power control methods were improved with the user spectrum allocation, power management, scheduling, and cooperative optimum downlink beamforming in [10]-[12] for the multi-cell environment of wireless networking.

Most of the current researchers focus on massive MIMO beamforming, association link and interference to noise ratio management to overcome the congestion through load balancing in the wireless network. But, the massive MIMO approach in the cellular and wireless network that is based on antenna array arrangement. On the other hand, the association link is more beneficial to improve the user link connectivity and data transmission. The interference to noise ratio controlling is mostly focused on managing the inter-cell interferences and transmission power.

To overcome the load balancing and congestion control over beamforming, this paper proposes an energy-efficient load management with multi-users handling scheme for emerging wireless networks. The strategy is divided into three steps. Firstly, beamforming transmission power breath-in and 
breath-out according to user location is employed to manage energy efficient based load management. Secondly, for load balance by handling multi-users data within the single beamforming transmission with the help of data multiplexing gain characteristics in which the various users can send the data simultaneously to improve overall network capacity. Finally, the multiple user data are extracted from the multiplexed transmission by MMSE/ZF decoders. The discussed strategy is useful to conquer the load balancing, congestion control, inter-cell interferences, energy-efficient data transmissions and it can also increase the number of users within a single beamforming transmission for emerging wireless network.

These residual sections are organized as follows. Section II explains the system model. Section III describes the MIMO with beamforming for load balancing. The methodology of Beam breathing based load management scheme is briefly explained in Section IV. A comparative simulation results between beam breathing and cell zooming are given in Section V and finally conclusion is drawn in Section VI.

\section{SySTEM MODEL}

The system design is based on multi-users in a multi-cells system that contains $C$ cells. All cells are equipped with BTS to manage the beamforming transmissions and $A_{c}$ MU's, $A_{c} \geq 1$ and $c=1,2, \ldots, C$. The total numbers of MU's in the system are defined as $A=\sum_{c=1}^{C} A_{c}$. We assume that each BTS are equipped with an equal number of antennas, defined as $M_{D}$ $\geq 1$. The overall antennas around all BTS's are defined as $M=$ $M_{D} C$. At receiver site, we assume that every $A$ MU's is equipped with $N$ antennas, $N \geq 1$. It is also assumed that the BTSs with beamforming downlink transmission are based on a flat frequency response for all propagation channels. Let, $S_{i, j}$ $S_{i, j}=\sum_{i=1}^{A} x_{i}$ is defined as the sum of all multi-users data symbols for the $i^{\text {th }}$ and $j^{\text {th }}$ users which are then weighted by the downlink beamforming weights. The beamforming downlink weights corresponding to different active and align MU's, which can regulate the BTS transmission and downlink signal energy, where they jointly computed according to the channel state information as shown in Fig. 1. Whereas, we assumed that the user location are well identified at all BTS's and also shared amongst neighbor's BTS by scheduling mechanism, and it is also considered that the $i^{\text {th }}$ and $j^{\text {th }}$ users are located in the same direction of downlink beamforming transmission. BTS's are also synchronized for the Coordinated Multi-Point (CoMP) processing of downlink data transmissions [13]. Here, for simplicity, the single BTS transmitted broadband signal received by the active MU $Z_{i . j}$ is represented as

$$
Z_{i, j}=\sum \mathbf{h}_{i, j}^{H} w S_{i, j}+\sum_{\substack{m \neq i, n \\ m \neq j}} \mathbf{h}_{m, n}^{H} w S_{m, n}+g_{i, j}
$$

where the $w \in \mathbb{C}^{M \times 1}$ is considered as beamforming vector, $S_{i, j}$ are symbolized as information signal at $i^{\text {th }}$ and $\mathrm{j}^{\text {th }} \mathrm{UM}$ 's , respectively; the $\mathbf{h}_{i, j} \in \mathbb{C}^{N \times M}$ is defined as downlink channel between from $M$ BTS's antennas to $i^{\text {th }}$ and $\mathrm{j}^{\text {th }}$ MU's, and $g_{i, j} \in \mathbb{C}^{N \times 1}$ denotes the receiver noise. Assume that $g_{i, j} \sim C N\left(0, \sigma_{i, j}^{2}\right), \forall_{i, j}$. Let's assume that the weight vector $w$ has been gathered from perfect channel state information (CSI) signaling. The beamforming transmission steers MU's according to power allocations and weight vector, so that the desired SINR at each mobile user is overhead to the objective standards. Whereas the transmission power is considered according to the edge/far distance users to cover both near and far users in single beamforming transmissions.

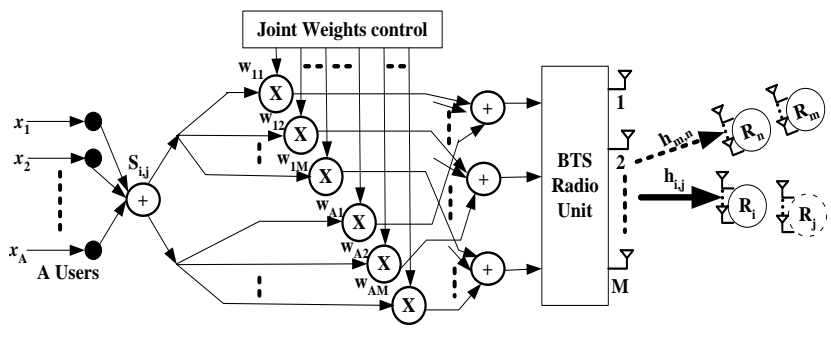

Fig. 1. System model.

The signal-to-noise-plus-interference ratio (SNIR) at the $i^{\text {th }}$ receivers end is defined as

$$
\operatorname{SINR}_{i}=\frac{\left|w^{H} h_{i}\right|^{2}}{\sum_{m \neq i, n}\left|w^{H} h_{m, n}\right|^{2}+\sigma^{2}}
$$

The beamforming transmission power concords according to edge/far $j^{\text {th }}$ user.

$$
\operatorname{SINR}_{j}=\frac{\left|w^{H} h_{j}\right|^{2}}{\sum_{m \neq j, n}\left|w^{H} h_{m, n}\right|^{2}+\sigma^{2}}
$$

The total power of transmission for breath-out to manage neighbor load balancing can be formulated as

$$
\begin{aligned}
& \max \left|w^{H} h_{i}\right|^{2}+\left|w^{H} h_{j}\right|^{2} \\
& \text { s.t. } S N I R_{j} \geq S N I R_{i}, \forall i, j=1,2, \ldots N
\end{aligned}
$$

The total transmission power of breath-in for congestion control can be expressed as

$$
\begin{aligned}
& \min \left|w^{H} h_{i}\right|^{2}+\left|w^{H} h_{j}\right|^{2} \\
& \text { s.t. } S N I R_{j} \geq \operatorname{SNIR}_{i}, \forall i, j=1,2, \ldots N
\end{aligned}
$$

The load balancing is achieved by handing-over the user to neighbor beamforming regions to optimize the performance of the wireless network. The instant bandwidth effectiveness $e_{i j}(\tau)$ of $j^{\text {th }}$ user in beam region $k^{\text {th }}$ BTS at $\tau$ packets frame with given SNIR is represented as

$$
e_{j, k}(\tau)=\log _{2}\left[1+\operatorname{SINR}_{j, k}(\tau)\right]
$$

As load balance is a periodical manner with an extended 
time range, therefore the load occupied by user $j^{\text {th }}$ in the beam region $k^{\text {th }}$ BTS at time $t$ is signified as

$$
\rho_{j . k}(t)=f\left(e_{j, k}(t)\right)
$$

where $f($.) denotes the mapping relation between the occupied load and the bandwidth efficiency of user $j^{\text {th }}$ in the beam $k^{\text {th }}$ BTS. Therefore, the total load $\rho_{j}(\mathrm{t})$ of beam region $\mathrm{j}^{\text {th }}$ at time $t$ is:

$$
\rho_{j}(t)=\sum_{k \in K_{i}} \rho_{j, k}(t)
$$

The MIMO recursive detection techniques such as (MMSE and $\mathrm{ZF}$ ) decoders with successive interference cancellers (SIC) are used to extract the relevant information from noisy signals. The detail structures of ZF and MMSE equalizers with SIC are well studied in [14], [15]. While, The zero forcing method efforts to find a matrix $(W)$ to fulfill the $(W \mathrm{H}=$ 1). ZF decoding linearity can be expressed as

$$
W=\left(H^{\mathrm{H}} H\right)^{-1} H^{\mathrm{H}}
$$

The MMSE methodology struggles to obtain the coefficient matrix $(W)$, which diminishes the condition

$$
E\left\{\left[W_{Z-S}\right]\left[W_{Z-S}\right]^{\mathrm{H}}\right\}
$$

According to the Tikhonov regularization [16], we can express as

$$
W=\left[H^{\mathrm{H}} H+N_{o} I\right]^{-1} H^{\mathrm{H}}
$$

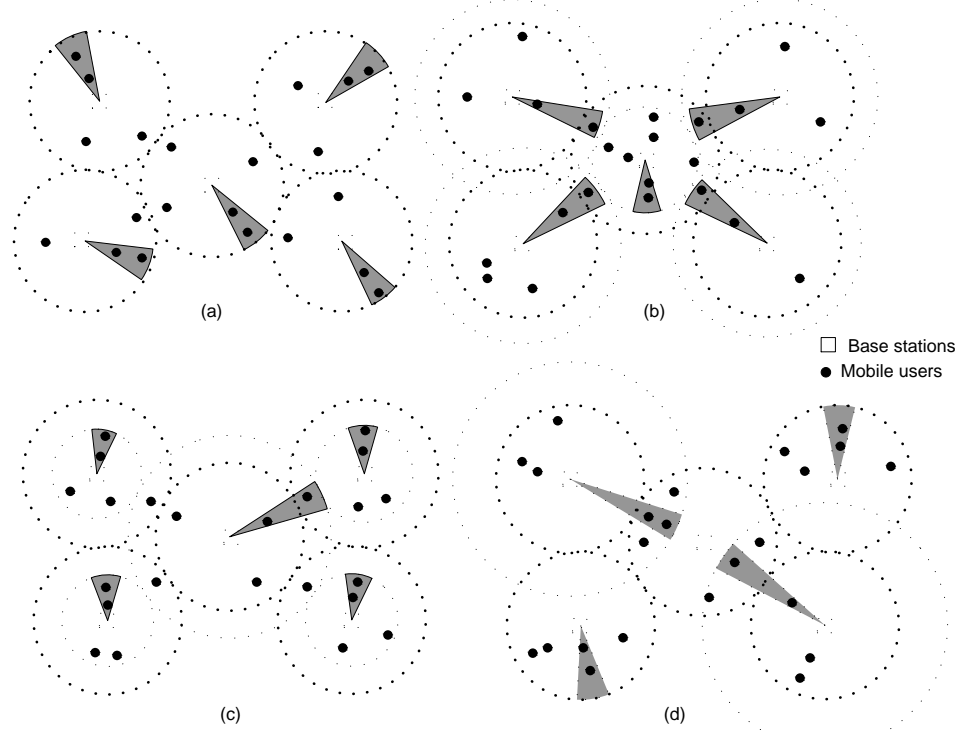

Fig. 2. Beam zooming procedure in cellular phone networks: a) Single beam multi-users model; b) Congestion control by central beamforming region zoom-in; c) Central region zoom-out to decrease neighboring areas load; d) Neighboring area zoom-out load manage and central transmitter sleep.

\section{MIMO WITH BEAMFORMING FOR LOAD BALANCING}

The multi-users MIMO (MU-MIMO) has the ability of augmented diversity, elevated capacity, considerable spatial gain and interfering repression [17]. There have been plenty of schemes conceiving such issues in wireless and cellular networks with the help of MIMO diversity and beamforming transmission; Even the beamforming with MIMO spatial and multiplex gain is useful to improve throughput and the number of users in emerging wireless networks. Intensive work was carried out to enhance the performance of overall wireless network [18]. One of them is load balancing.

The beamforming technique has been studied to overcome these limitations and issues. Consequently, the performance of a conventional cell zooming network can be significantly improved whenever beamforming is enabled across the different BTS's to improve the overall network's performance of future networks [19]. The beam range and capacity are generally set according to the estimation of maximum traffic weight during network optimization.

Nevertheless, the user's mobility and bursty nature of numerous data applications can have considerable spatial and temporal variations in traffic load of the cellular network. For example, the traffic load is heavy in offices during the daytime rather than night. While on the other hand, in residential areas, a contrary situation occurs in the evening time. In this situation, the static traffic load implementation is not optimal to manage the traffic load oscillation for cellular networks. Furthermore, the traffic variation is getting serious threat for future networks, as the next generation networks are shifting toward smaller coverage area to get optimum performance in heavy and fluctuated traffic [20].

\section{BEAM BREATHING BASEd LOAD MANAGEMENT SCHEME}

Beamforming (Beam) breath has the capability to make equilibrium in the traffic burden and minimizes the energy consumption. To explore the congestion control, a novel scheme of beam breathing is proposed; in which adaptively aligns the beam size concording to traffic consideration and user location. The active user location and direction can be identified by scheduling the channel for the user with the best instantaneous CSI. It is assumed that the entire user's CSI should be accessible at the BTS's to achieve the benefit of multiuser diversity. The CSI information of all active users simultaneously sends a response message to the BTSs following a broadcast probing message from center regions to 
neighbor's region BTSs and vice versa.

The exemplary of proposed beam breathing and single beam multi-users is demonstrated in Fig. 2. It is a typical cellular system model with four different beamforming regions. BTSs are placed in the center of each area; MU's are haphazardly spread within the beamforming regions, which are defined as solid black dots. The dotted circle symbolizes the beamforming signal strength regions and solid circles represent the beams breath-in and breath-out regions. The sum of multiple user data is transmitted by downlink beamforming transmission to avail the single beamforming transmission for multi-users, simultaneously. While, the total power of transmission for breath-out to manage neighbor load balancing can be formulated as

$$
\begin{aligned}
& \max \left|w^{H} h_{i}\right|^{2}+\left|w^{H} h_{j}\right|^{2} \\
& \text { s.t. } S N I R_{j} \geq S N I R_{i}, \forall i, j=1,2, \ldots N
\end{aligned}
$$

And, the total transmission power of breath-in for congestion control can be expressed as

$$
\begin{aligned}
& \min \left|w^{H} h_{i}\right|^{2}+\left|w^{H} h_{j}\right|^{2} \\
& \text { s.t. } \operatorname{SNIR}_{j} \geq \operatorname{SNIR}_{i}, \forall i, j=1,2, \ldots N
\end{aligned}
$$

The receivers are equipped with recursive detection techniques (ZF and MMSE equalizers with SIC) to retrieve the relevant information from a noisy channel. The each user extracts the data stream from the sum of multiple users by subtracting other user's information with the help of weightage and CSI control information.

The following cases are used to manage the load balancing in cellular networks and wireless network.

\section{A. All BTS Turned ON without Any Load Balancing}

In this case, all BTS are turned ON with traditional adaptive beamforming scheme as shown in (Fig. 2a). The beam breathing in/out and load sharing is not activated. While, the single beam multi-users handling scheme is employed to manage congestion control of multi-users management, simultaneously.

\section{B. Central Beam Zoom-in and Neighbor Beam Zoom-out to Manage the Load Balancing}

Whenever, several MU's shifts into the central beam region, it causes central region overloaded. To avoid the congestion in the central beam regions; The central beam can breath-in to minimize the power consumptions and neighbor's beam can breath-out for edge users to manage the load balancing as shown in (Fig. 2b). The benefits of dividing the load of central beam regions edge users among the neighbor's regions are to spread the power usage amongst the neighbor's regions. There is a condition that is employed for edge users of central beam regions; if the neighbor's regions have any own user in the same direction of the central region edge user to entertain, then it will enhance the own beam energy up-to far distance, in which "single-beam multi-users" is employed by summing multiple user's information in single downlink beamforming to serve simultaneously both own and the central region users to manage load balancing in the central region.

\section{Central Beam Zoom-out and Neighbor Beam Zoom-in to Manage the Load Balancing}

On the other hand, when several MU's moves-in from the central beamforming area into the neighbor beamforming regions to cause overloading, neighbor beamforming can breath-in and the central beamforming breath-out to prevent any probable coverage gaps (Fig. 2c). A condition is also applied here in which the users of both central and neighbor's regions should be located in the same direction. If the condition gets true, then the steering beam of the central region will breath-out with "single-beam multi-users" to accommodate the both central and neighbor's users, simultaneously. The benefits of sharing users' loads with central beam region to minimize the congestion of neighbor regions and efficient utilization of the central region beam transmission.

\section{Central BTS Sleep Mode and Neighbor Beam Zoom-out to Manage the Load Balancing}

Furthermore, the central region BTS can also go to sleep mode as shown in (Fig. 1d) to minimize the energy consumption. In this instance, the neighbor beamforming will breath-out to handle the MU's of central beam regions. This scenario is designed as a maximum capacity planning; in which the breath-in is not required anymore. Here, it is assumed that the central region is in sleep mode due to fewer MU's, central region MU's can be handled by neighbor's beam transmissions with the help of "single-beam multi-users".

Step1: Initialization: Overloaded region beamforming Zoom-in/Zoom-out

Step2: Power minimizes/ maximizes

a. Power minimizes to avoid congestion

$$
\begin{aligned}
& \min \left|w^{H} h_{i}\right|^{2}+\left|w^{H} h_{j}\right|^{2} \\
& \text { s.t. } \operatorname{SNIR}_{j} \geq \operatorname{SNIR}_{i}, \forall i, j=1,2, \ldots N
\end{aligned}
$$

b. Power maximizes to manage users load balancing

$$
\begin{aligned}
& \max \left|w^{H} h_{i}\right|^{2}+\left|w^{H} h_{j}\right|^{2} \\
& \text { s.t. } \operatorname{SNIR}_{j} \geq \operatorname{SNIR}_{i}, \forall i, j=1,2, \ldots N
\end{aligned}
$$

if more than one users are located in the same direction, then activate single beam multi-users handling mechanism with maximized power

else Single user downlink beamforming transmission with maximum power will manage the load balancing

Whenever, center or neighbor beam breath-in/breath-out to balance a load of the congested region, vice versa; the single beam multi-users technique is useful to efficiently handle the congestion by serving multi-users simultaneously in the network. The benefits of the proposed solution are to manage the congestion, increase the throughput by simultaneously handling the multiple users and reduce the energy consumption (by breath in-out and sleep mode) in wireless \& cellular networks.

The power consumption of sleep mode, BTS ON/OFF 
mode and active mode for each BTS is expressed as

$$
P(t)=P_{\mathrm{SM}}+P_{\mathrm{o}}+P_{T} f(t)
$$

where $P_{S M}$ is normalized to sleep mode power consumption, $P_{o}$ denotes the power efficiency required for an active BTS without traffic load, the power consumption with the traffic of single unit is represented as $P_{T}$ and the peak hour traffic is standardized as $f(t)$.

Let, $f(t)$ represent the everyday traffic flow pattern in a beam region, for example the traffic strength as a function of time $t$ by $t \in[0, T], T=24 \mathrm{~h}$ and $t=0$ peak hours;

And the earliest time instant is defined as $\tau$. The detail derivations of energy consumption are mentioned in [21].

The energy consumed during all BTS ON is

$$
\begin{aligned}
E E_{\text {ALLON }} & =\int_{0}^{T}\left[P_{S M}+P_{o}+P_{T} f(t)\right] d t \\
& =T\left(P_{S M}+P_{o}\right)+P_{T} \int_{0}^{T} f(t) d t
\end{aligned}
$$

Energy consumed during BTS sleep mode is expressed as

$$
\begin{aligned}
E E_{\text {SLEEP }}= & 2 \int_{0}^{\tau}\left[P_{S M}+P_{o}+P_{T} f(t)\right] d t \\
& +2(T / 2-\tau) P_{S M} \\
& =T P_{S M}+2 \tau P_{o}+2 P_{T} \int_{0}^{\tau} f(t) d t
\end{aligned}
$$

The power consumed during BTS ON/OFF according to traffic (Low /High) conditions is expressed as

$$
\begin{aligned}
E E_{\text {ON } / O F F} & =2 \int_{0}^{\tau}\left[P_{S M}+P_{o}+P_{T} f(t)\right] d t \\
& +2 \int_{\tau}^{T / 2}\left[P_{S M}+P_{o}+P_{T} \frac{1}{x} f(t)\right] d t \\
& =T\left(P_{S M}+P_{o}\right)+2 P_{T} \int_{0}^{\tau} f(t) d t \\
& +2 P_{T} \frac{1}{x} \int_{\tau}^{T / 2} f(t) d t
\end{aligned}
$$

The combination of beam breathing and single beam multi-users handling schemes are useful to enhance the capability of load management and energy efficiency. Furthermore, the beam breathing technique is helpful to manage the congestion and energy consumption through breath-in/out and sleep mode for load balancing and reduce energy consumption. While, Single beam multi-users handling is also competent to manage congestions and energy consumptions through the single beam transmissions for multi-users data handling simultaneously.

\section{Simulation AND RESUlt Discussion}

To validate the effectiveness of the proposed scheme, some simulation results are presented. The simulation outline is situated with five BTS within four different regions. It assumes that the BTS can inflate the coverage up-to 50\% of the precise range. The path-loss is considered for the flat fading channel among BTS's and MU's granting to ITU standards [22]. Energy utilization is $400 \mathrm{~W}$ for BTS in active mode and $10 \mathrm{~W}$ in sleep mode with $5 \mathrm{MHz}$ bandwidth of every BS. The transmitter and receiver gains are adjusted to $20 \mathrm{~dB}$ and $1 \mathrm{~dB}$ respectively. The data rate is adjusted around 125 Mbps. The receivers are equipped with MMSE and ZF equalizers to retrieve desired information. The overall simulation duration is set to be 1 hour.

The scenario of the beam breathing scheme is discussed below.

1) All ON BTS (AOB): All BTS are turned ON. Beam zoom-in/out is unavailable, Congestion control by single beamforming multiuser handling.

2) Central beam breath-in (CBBI): All BTS's are turned ON. The central beam breath-in is to manage congestion and neighboring beam breath-out to efficiently manage the load balancing.

3) Central beam breath-out (CBBO): All BTS's are turned $\mathrm{ON}$. The central beam breath-out is to efficiently manage the load balancing and neighboring beam breath-in for congestion control.

4) Neighboring beam breath-out (NBBO): 4 BTS's are turned ON. The central BTS is in sleep mode and the neighbor's beam breath-out is to efficiently manage the load balancing.

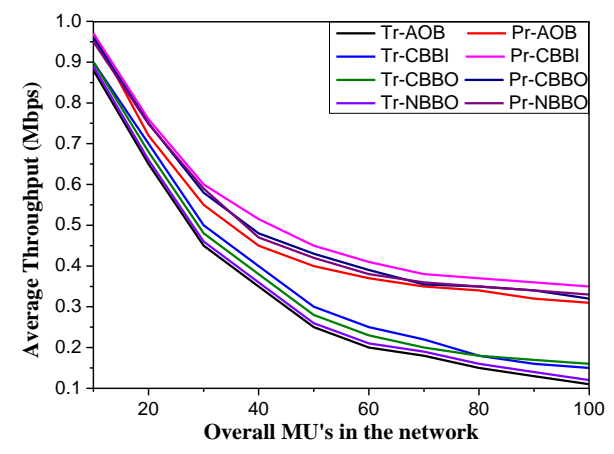

Fig. 3. Overall average throughput.

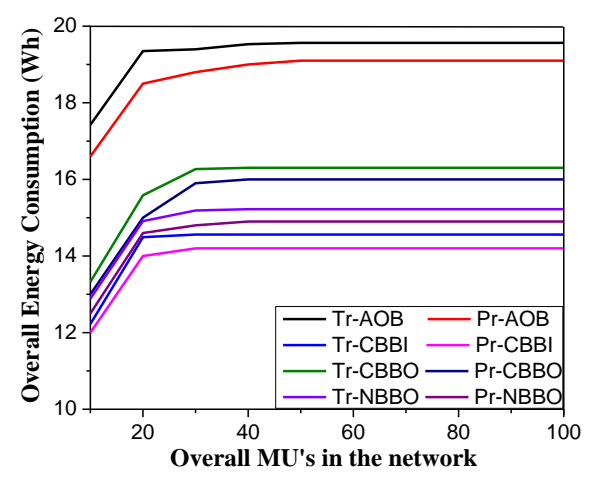

Fig. 4. Overall energy consumption.

Here, the QoS performance of the proposed scheme $(\mathrm{Pr})$ is compared with traditional cell zooming (Tr) network. It is observed from the simulation results that the scenarios of proposed beam breath-in/out load sharing schemes (AOB, CBBI, CBBO and NBBO) have better performance than the traditional cell zooming schemes due to multi-users handling 
by single beamforming transmission. Moreover, there is also reduce in the inter cell's interferences and better power consumptions. The comparisons between 4 different scenarios of the proposed scheme are also evaluated in this simulation. It is shown in Fig. 3 which reveals that Pr-CBBI, Pr-CBBO, and Pr-NBBO have overall better performance around $20 \%$ with minor difference than each other's due to less interference and better energy efficient load balancing.

Subsequently, the cause of QoS degradation in other scenarios looks individually; Pr-AOB performance is a bit degraded because the edge MU's was unable to handle properly due to unavailability of load balancing to control congestions.

The energy efficiency of each scenario is briefly explained in Fig. 4. It clearly shows that the proposed method has better energy efficiency than traditional cell zooming scheme (around $15 \%$ ). This is because the proposed beam breathing scheme has the ability of user steering beamforming with multi-users handling to serve the users energy efficiently.

The comparison of the energy consumption between the proposed beam breathing scenario schemes (Pr-AOB, Pr-CBBI, Pr-CBBO and Pr-NBBO) shows that the Pr-CBBI, Pr-CBBO and Pr-NBBO have better energy efficiency than Pr-AOB. This is because of breath-in/out and sleep mode, divide the power and user load into the central and neighboring regions. When we look in more depth, it can be observed that the Pr-CBBO consumes bit higher energy than Pr-CBBI and Pr-NBBO. This is due to neighbors beam breath-in and central beam breath-out to manage the neighbor users' load. The AOB has relatively higher power consumption than other cases due to all BTS ON.
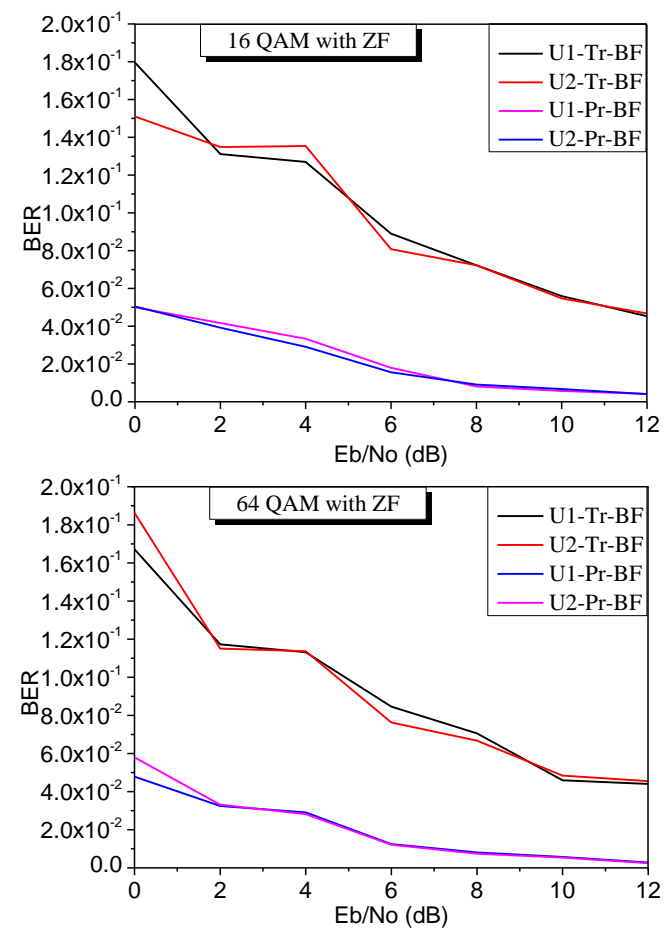

Fig. 5. ZF with 16QAM \& 64QAM.

The proposed scheme enhanced the throughput around $20 \%$ and decreased almost $15 \%$ of energy consumptions. The QoS performance is enhanced due to user steer ability of beamforming, in which transmission is directly projected to data delivering users to avoid the energy wastage, Multi-users handling in simultaneous transmission and avoid other cells interferences.

The results of the single beam multi-users scheme are discussed in this section. Furthermore, it is divided into two more steps.

\section{A. $16 \& 64$ QAM over $Z F$}

The simulation results of ZF over $16 \& 64$ QAM constellations are discussed. Whereas, the bit error rate (BER) is calculated against the signal to noise ratio (SNR) to evaluate the comparison between the proposed beamforming (Pr-BF) and traditional beamforming (Tr-BF) schemes. It can be observed from Fig. 5 that BER of Pr-BF is lesser than that of Tr-BF from start to end, and it is decreased gradually upto $12(\mathrm{~dB})$ with the same situation. It is noticeable from the results that the Pr-BF is superior to Tr-BF method. Furthermore, it is elaborated that the proposed scheme achieves the key task in which the single beamforming smoothly transit multi-users data simultaneously with better performance.
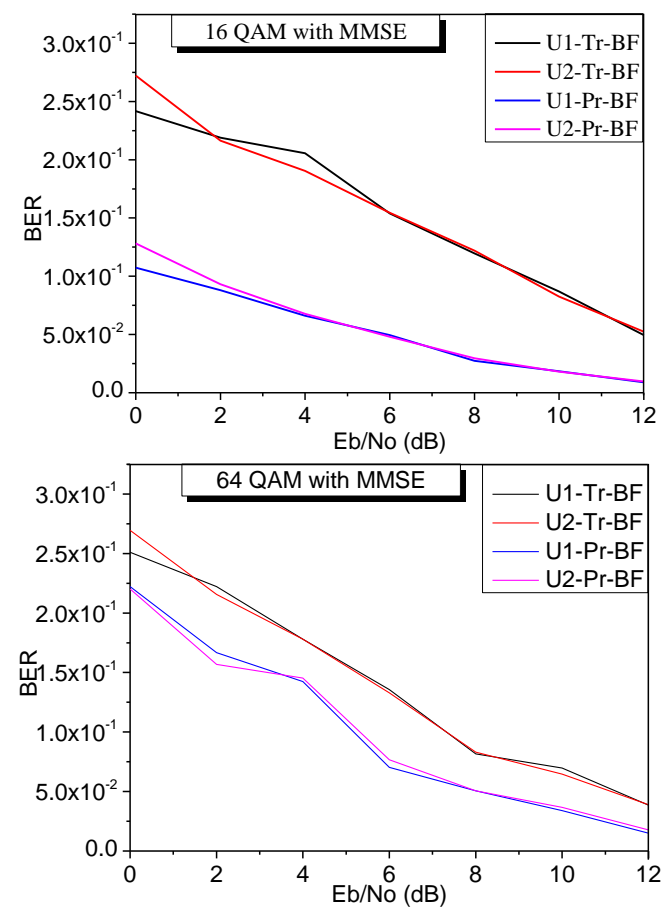

Fig. 6. MMSE-with-16QAM-\&-64QAM.

\section{B. $16 \& 64$ QAM over MMSE}

The simulation is conducted with MMSE equalizer for Pr-BF and Tr-BF method with 16-QAM and 64-QAM modulations. The simulation results are compared between Pr-BF and Tr-BF schemes for MMSE over 16 \& 64 QAM modulations, and evaluated the BER performance versus SNR in Fig. 6. Overall less BER of proposed scheme shows that it is much better than the traditional schemes. Moreover, it can also be seen that the single beamforming transmission can smoothly handle the multi-users information by the proposed scheme. It is clearly noticeable from simulation results that the Pr-FB techniques are superior in both cases (ZF and MMSE) equalizer than traditional transmission due to simultaneous smooth transmission of multi-users with the help of spatial multiplexing gain. The traditional schemes 
(Tr-BF) are based on multi-layer beamforming for multi-users handling that consumes extra processing time which effect on BER by delay processing. The Pr-BF has the ability of simultaneous transmission of multi-users with less BER by single layer transmission.

\section{CONCLUSION}

In this paper, a beam breathing based energy efficient load managing scheme for emerging wireless networks is proposed. The proposed method is based on adaptive beamforming (breath-in/out) for power control and multiplexed gain transmission. The multiplexed MU's data can be recovered from spatially multiplexed transmission by linear equalizers like MMSE and ZF equalizers. In addition, the single layer multi-users transmission scheme is effective to increase the number of users and improve system performance by reducing BER in beamforming transmission. The traditional cell zooming scheme can be upgraded into proposed beam breathing systems to improve the load management and energy consumption. It is illustrated by simulation results that the proposed scheme is quite useful to decrease the energy consumption and congestion, and also constructive to increase overall network throughputs.

\section{REFERENCES}

[1] H. Dahrouj and Y. Wei, "Coordinated beamforming for the multicell multi-antenna wireless system," IEEE Trans. on Wireless Communications, vol. 9, no. 5, pp. 1748-1759, May 2010.

[2] L. Long Bao, "QoS-aware BS switching and cell zooming design for OFDMA green cellular networks," in Proc. Global Communications Conference (GLOBECOM), 2012, pp. 1544-1549.

[3] S. N. Khan Marwat, S. Meyer, T. Weerawardane, and C. Goerg, "Congestion-aware handover in LTE systems for load balancing in transport network," ETRI Journal, vol. 36, no. 5, pp. 761-771, Oct. 2014.

[4] K. Shen and W. Yu, "Distributed pricing-based user association for downlink heterogeneous cellular networks," IEEE Journal on Selected Areas in Communications, vol. 32, no. 6, pp. 1100-1113, June 2014.

[5] T. S. Kang and B. W. Seo, "Joint optimization of user set selection and transmit power allocation for orthogonal random beamforming in multiuser MIMO systems," ETRI Journal, vol. 34, no. 6, pp. 879-884, Dec. 2012.

[6] N. Gresset et al., "Interference-avoidance techniques: Improving ubiquitous user experience," IEEE Vehicular Technology Magazine, vol. 7, no. 4, pp.37-45, Dec. 2012.

[7] Y. Li et al., "Dynamic beamforming for three-dimensional MIMO technique in LTE-advanced networks," International Journal of Antennas and Propagation, vol. 2013, Article ID 764507, 8 pages, 2013.

[8] W. Lee et al., "Downlink vertical beamforming designs for active antenna systems," IEEE Transactions on Communications, vol. 62, no. 6, pp. 1897-1907, Jun. 2014.

[9] Y. Huang, C. W. Tan, and B. D. Rao, "Joint beamforming and power control in coordinated multicell: Max-min duality, effective network and large system transition," IEEE Transactions on Wireless Communications, vol. 12, no. 6, pp. 2730-2742, Jun. 2013.

[10] R. Stridh, M. Bengtsson, and B. Ottersten, "System evaluation of optimal downlink beamforming with congestion control in wireless communication," IEEE Trans. on Wireless Communications, vol. 5, no. 4, pp. 743-751, April 2006.

[11] J. K. Hye and G. K. Chung, "A distributed implementation of mobility load balancing with power control and cell reselection in 4G network," International Journal of Network Management, vol. 24, no. 5, pp. 337-356, Sept/Oct. 2014

[12] W. Feng, S. L. Feng, Y. Zh. Zhang, and X. W. Xia, "Cross-layer resource allocation in multi-interface multi-channel wireless multi-hop networks," ETRI Journal, vol. 36, no. 6, pp. 960-967, Dec. 2014.
[13] C. Yong, M. Pesavento, and A. Philipp, "Joint network optimization and downlink beamforming for CoMP Transmissions using mixed integer conic programming," IEEE Transactions on Signal Processing, vol. 61, no. 16, pp. 3972-3987, Aug. 2013.

[14] N. I. Miridakis and D. D. Vergados, "Efficient detection-switching based on successive interference cancellation for multiple-input-multiple-output orthogonal frequency division multiplexing systems," Signal Processing, IET, vol. 7, no. 5, pp. 436-443, July 2013.

[15] A. H. Mehana and A. Nosratinia, "Diversity of MMSE MIMO receivers," IEEE Trans on Information Theory, vol. 58, no. 11, pp. 6788-6805, Jun 2012.

[16] L. Hu, X. Y. Zhou, and L. W. Zhang, "Blind multiuser detection based on Tikhonov regularization," Communications Letters, vol. 15, no. 5, pp. 482-484, May 2011.

[17] X. Li et al., "Cooperative communications based on rate-less network coding in distributed MIMO systems [Coordinated and Distributed MIMO]," IEEE Wireless Communications, vol. 17, no. 3, pp. 60-67, Jun. 2010.

[18] D. Gesbert et al., "Multi-cell MIMO cooperative networks: a new look at interference," IEEE Journal on Selected Areas in Communications, vol. 28, no. 9, pp. 1380-1408, 2010.

[19] Z. Niu et al., "Cell zooming for cost-efficient green cellular networks," IEEE Communications Magazine, vol. 48, no. 11, pp. 74-79, Nov. 2010.

[20] Z. Zhang, K. C. The, and K. H. Li, "A semidefinite relaxation approach for beamforming in cooperative clustered multicell systems with novel limited feedback scheme," IEEE Transactions on Vehicular Technology, vol. 63, no. 4, pp. 1740-1748, May 2014.

[21] M. A. Marsan et al., "On the effectiveness of single and multiple base station sleep modes in cellular networks", Computer Networks, vol. 57, Issue 17, pp. 3276-3290, December 2013.

[22] 3GPP TR 36.814 V1.2.1, Further Advancements for EUTRA: Physical Layer Aspects, Jun. 2009.

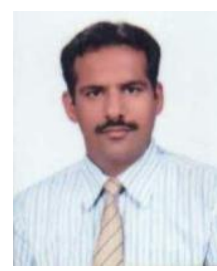

Naveed Ali Kaim Khani is a candidate of Ph.D. degree at School of Communication and information engineering, Dalian University of Technology, Dalian, China. He has a bachelor degree in electronics engineering from Sir Syed University of Engineering and Technology, Karachi, Pakistan and masters degree in computer and communication networks from Telecom \& Management SudParis (ex INT), Evry, France. He has worked as lecturer \& Head of network department (from 2007 to 2009 at Universities of Engineering Science and Technology of Pakistan (UESTP-France), Karachi, Pakistan and also work as an assistant professor from 2009 to 2011 at COMSATS Institute of information Technology, Abbottabad, Pakistan. His main research areas include, MIMO, Beamforming, WiMax over GSM/GPRS Network, a Physical layer of Wireless Network, Energy Efficient Wireless network.

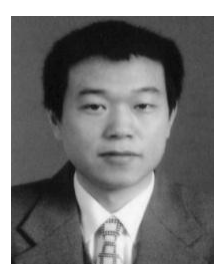

Zhe Chen received his B.S degree in electronics engineering, the M.S degree in signal and information processing, and the Ph.D. Degree in signal and information processing from Dalian university of Technology (DUT), Dalian, China, in 1996, 1999 and 2003 respectively. he joined the Department of electronics Engineering, DUT, as a lecturer in 2002, and become an associate professor in 2006. His research includes speech processing, Image processing, and wideband wireless communication.

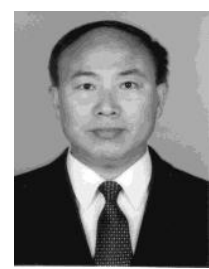

Fuliang Yin was born in Fushun city, Liaoning province, China, in 1962. He received the B.S. degree in electronic engineering and M.S. degree in communications and electronic systems from Dalian University of Technology (DUT), Dalian, China, in 1984 and 1987, respectively. He joined the Department of Electronic Engineering, DUT, as a lecturer in 1987 and became an Associate Professor in 1991. He has been a Professor at DUT since 1994, and the dean of the School of Electronic and Information Engineering of DUT from 2000 to 2009. His research interests include digital signal processing, speech processing, image processing and broadband wireless communication. 